\title{
El desafío de la segregación escolar en República Dominicana
}

\section{Un estudio por nivel socioeconómico en el nivel de primaria}

\author{
F. Javier Murillo* | Sandra Carrillo***
}

El objetivo es estimar la magnitud de la segregación escolar por nivel socioeconómico en educación primaria en República Dominicana, con una mirada específica en las escuelas urbanas. Se realiza una explotación especial de la Evaluación Diagnóstica Nacional del 2017 que evalúa a todos los estudiantes de tercer grado de primaria. Se analizan 157,754 alumnos matriculados en 7,791 escuelas ubicadas en las 18 Direcciones Regionales Educativas en las que se organiza el sistema educativo dominicano. Se utilizan los índices de Gorard y de Aislamiento, donde la variable criterio es el índice socioeconómico, para cuatro grupos minoritarios. Los resultados indican que la magnitud de la segregación escolar en las escuelas de primaria es medio-alta, con una tendencia a una mayor segregación de los estudiantes con familias de mayor nivel socioeconómico. Se evidencia la necesidad de reducir la segregación escolar para acortar las desigualdades sociales del sistema educativo del país.

The main goal of the present article is to estimate the magnitude of school segregation according to the students' socioeconomic status in primary education in the Dominican Republic; specifically in schools situated in the urban areas. In order to do this, we exploited the information provided by the National Diagnostic Assessment 2017, which assesses all third-grade primary school students. Our study analyzes the cases of 157,754 students enrolled in 7,791 schools located in the 18 Regional Educational Directorates in which the Dominican educational system is divided. We also used the Gorard and Isolation indexes with the socioeconomic index as the criterion variable, for four minority groups. The results indicate that the magnitude of school segregation in primary schools is medium-high, showing that students from families with a higher socioeconomic status tend to exercise more segregation than others. It becomes evident that we need to reduce school segregation in order to shorten the social inequalities of the country's educational system.
Palabras clave

Equidad educativa

Nivel socioeconómico

Educación básica

Desigualdad social

Segregación escolar

Keywords

Educational equity

Socioeconomic status

Basic education

Social inequality

School segregation

Recepción: 22 de agosto de 2019 | Aceptación: 24 de junio de 2021

DOI: https://doi.org/10.22201/iisue.24486167e.2021.174.59552

* Director de la Cátedra UNESCO en Educación para la Justicia Social y profesor titular de la Universidad Autónoma de Madrid (UAM) (España). Doctor en Ciencias de la Educación. Líneas de investigación: educación para la justicia social; segregación escolar; calidad y equidad de la educación. Publicaciones recientes: (2021, en coautoría con G. Belavi), "Differential Impact of School Segregation in the Performance of Native and Non-Native Students in Spain", Journal of New Approaches in Educational Research, vol. 10, núm. 1, pp. 85-100. DOI: https://doi.org/10.7821/ naer.2021.1.559; (2021, en coautoría con A. Almazán y C. Martínez-Garrido), "La elección de centro educativo en un sistema de cuasi-mercado escolar mediado por el programa de bilingüismo", Revista Complutense de Educación, vol. 32, núm. 1, pp. 89-97. DOI: https://doi.org/10.5209/rced.68068.CE: javier.murillo@uam.es. ORCID: orcid. org $/ 0000-0002-8003-4133$

** Investigadora principal del Instituto de Estudios Peruanos (IEP) (Perú). Doctoranda en Educación. Línea de investigación: políticas educativas relacionadas con segregación escolar, género y desigualdades sociales. Publicaciones recientes: (2021, en coautoría con F.J. Murillo), "Incidencia de la segregación escolar por nivel socioeconómico en el rendimiento académico. Un estudio desde Perú", Archivos Analíticos de Politicas Educativas, vol. 29, núm. 49, pp. 1-24, en: https://epaa.asu.edu/ojs/article/view/5129; (2021, en coautoría con F.J. Murillo), "Segregación escolar por nivel socioeconómico en Colombia y sus departamentos", Magis, Revista Internacional de Investigación en Educación, vol. 14, pp. 1-23. DOI: https://doi.org/10.11144/Javeriana.m14.sens. CE: carrillo.sandra@pucp. pe. ORCID: https://orcid.org/0000-0003-2871-4702 


\section{INTRODUCCIÓN}

América Latina es una de las regiones más desiguales del planeta, y esto no sólo tiene que ver con la distribución de los ingresos, sino también con el desarrollo de capacidades (CEPAL, 2016). La desigualdad limita las posibilidades de grandes grupos de la población de acceder a más y mejor educación, así como de mejorar su calidad de vida y ascender socialmente para salir de la pobreza.

El Programa Internacional de Evaluación de Estudiantes (PISA) de la OECD muestra, sistemáticamente, los bajos resultados de los países de la región y los altos niveles de segregación escolar por nivel socioeconómico, comparados con el promedio del total de los países participantes (OECD, 2019). La segregación escolar, entendida como la distribución desigual de los alumnos en las escuelas según sus características personales o condiciones sociales, es un fenómeno que profundiza las brechas educativas y dificulta la igualdad de oportunidades.

República Dominicana no es la excepción. Si bien la educación primaria casi ha logrado llegar a la cobertura universal, las brechas en el acceso a los niveles de inicial y secundaria aún persisten a pesar de que estos tres niveles oficialmente son obligatorios y gratuitos (13 años). Adicionalmente, están las dificultades para lograr resultados de aprendizaje adecuados, que permitan no sólo mantenerse en la escuela, sino también concluirla a la edad correspondiente (SITEAL, 2021). ${ }^{1}$ Asimismo, República Dominicana es el país de la región que menos invirtió en educación pública entre los años 1990 y 2009; además, 24 por ciento de sus estudiantes está matriculado en escuelas privadas (Arcidiácono et al., 2014; Pereyra, 2008), situación que se explica, en parte, por las "dinámicas y mecanismos propios de una alianza público-privada histórica y del posterior desarrollo de la modalidad de escuelas privadas de bajo costo en ámbitos urbanos" (Verger et al.,
2017: 74). En este contexto, los más perjudicados han sido los estudiantes de bajos ingresos que viven en las zonas más alejadas.

El año 2010 marcó un hito histórico importante en la educación dominicana: la sociedad civil se movilizó para exigir al Estado garantizar el derecho a una educación digna. Es así que, desde el 2013, "el país ha venido aumentando su gasto público en educación (alrededor de 2 por ciento del PIB a 4 por ciento) y ha ido adoptando diversas medidas de política con el objetivo de mejorar los resultados del sistema educativo nacional" (Checo, 2019: 8). Sin embargo, la segregación escolar aún no se ha identificado como un problema del sistema educativo dominicano, ni se reconoce que atenta contra la equidad y calidad de éste. En ese sentido, este artículo busca aportar a la discusión al estimar la magnitud de la segregación escolar por nivel socioeconómico en República Dominicana en las escuelas del nivel primaria. Conocer la segregación escolar por nivel socioeconómico puede dar luces para enfocar la discusión en un tema que implica hacer reformas para acortar las desigualdades sociales del sistema educativo y aportar a la construcción de una sociedad más justa e inclusiva.

\section{REVISIÓN DE LA LITERATURA}

Si bien América Latina cuenta con una importante tradición histórica de estudios sobre inclusión y equidad educativa, no es sino hasta la primera década del presente siglo que el incremento de investigaciones sobre el tema se volvió significativamente importante (Plá, 2015). Esto se explica, en parte, por los modelos educativos implementados en la década de 1990, los cuales produjeron una mayor cobertura en la educación básica y obligatoria, pero generaron drásticas formas de desigualdad y exclusión educativa (Gentili, 2009).

Es así que la investigación educativa en la región se ha centrado principalmente en los

\footnotetext{
${ }^{1}$ Ver también UNESCO Institute for Statistics (UIS), en: http://data.uis.unesco.org (consulta: 20 de mayo de 2021).
} 
factores que explican los altos niveles de exclusión del sistema escolar, el bajo rendimiento académico y la notable inequidad educativa (Bonal y Bellei, 2018). Los artículos publicados en el último quinquenio sobre sistemas educativos y/o políticas educativas profundizan los temas relacionados con calidad de la educación, equidad, acceso a la educación, reforma escolar, segregación escolar, legislación educativa y políticas educativas públicas (Murillo y Martínez-Garrido, 2019). En esa misma línea, los estudios sobre segregación escolar - entendida como un aspecto particular de la noción más amplia de desigualdad social y de igualdad de oportunidades educativas - son bastante recientes en la región (no tienen más de dos décadas) y sehan ido incrementando, de manera sostenida, en el transcurso de los últimos años (Carrillo, 2020).

La segregación escolar se refiere a la distribución desigual o desbalance de los estudiantes en los centros educativos según sus características personales o condiciones sociales. Los primeros estudios sobre este fenómeno datan de la segunda mitad del siglo pasado, a raíz de la sentencia del Tribunal Supremo de Estados Unidos al declarar inconstitucionales las leyes estatales que establecían escuelas públicas separadas por color de piel. Esta sentencia del año 1954, sumada a la publicación del Informe Coleman (1966), convirtió las políticas de segregación y desagregación escolar en un objeto de estudio para académicos e investigadores, originalmente centradas en el origen étnico racial en Estados Unidos (Orfield, 2001; Reardon y Owens, 2014) y unos años después en el nivel socioeconómico (Dupriez, 2010; Gorard y Smith, 2004; Orfield y Lee, 2005) y en el origen nacional (Karsten, 2010; Murillo et al., 2017) de los estudiantes y sus familias, estos últimos con especial incidencia en Europa.

Sibien el fenómeno de la segregación es multidimensional, dos dimensiones han recibido mayor y especial atención en el campo educativo: la similitud o uniformidad (evenness) y la exposición (exposure). La similitud o uniformidad hace referencia a la tendencia de distintos grupos de estudiantes a distribuirse de manera desigual entre las escuelas (unidades organizativas); en ese sentido, se dice que un grupo está segregado si está sobrerrepresentado en algunas unidades (Gorard y Taylor, 2002). La dimensión de exposición, por su parte, se refiere a la probabilidad de interacción entre miembros de diferentes grupos o categorías sociales, es decir, el grado en que unos y otros están "expuestos" a personas de otros grupos; así, la segregación de un grupo se refiere al grado de aislamiento relativo de éste respecto de otras categorías sociales (Lieberson, 1981). Para medir estas dimensiones se utiliza un conjunto de índices; los más usados son el de Duncan, el de Gorard y la raíz cuadrada de Hutchens para medir la dimensión de uniformidad; y los índices de aislamiento y de interacción mutua para medir la dimensión de exposición (Murillo, 2016).

De esta manera, decimos que la segregación es un problema cuando las diferencias de esta distribución (el desbalance) afectan las probabilidades de interacción entre miembros de los diferentes grupos sociales; es decir, cuando surge la pregunta por el grado en que miembros de diferentes grupos o categorías sociales pueden llegar a interactuar, compartir ciertas experiencias o ser afectados por condiciones compartidas (Bellei, 2013; Gasparini et al., 2011). En otras palabras, cuando los estudiantes se agrupan en escuelas muy homogéneas interiormente, pero muy distintas entre sí (por su nivel socioeconómico, grupo étnico, procedencia, habilidades, etc.) y esa concentración afecta a determinados grupos (los segrega), lo que no es deseable ni compatible con los derechos humanos y la igualdad de oportunidades.

Esta evidente preocupación, asociada a la igualdad de oportunidades, tiene que ver con los efectos negativos de la segregación escolar en la calidad y riqueza de la experiencia formativa de los estudiantes. Los aspectos cívicos y de integración social, que en el largo plazo garantizan el "capital social" de un país, se desarrollan mejor en aulas que reflejan la diversidad 
de la sociedad misma, lo que a su vez favorece también la equidad y la calidad educativa relacionada al logro de los objetivos académicos y los aprendizajes. En ese sentido, las interacciones entre las personas que integran la escuela se convierten en recursos fundamentales de esta experiencia, principalmente si esta interacción se da entre estudiantes que provienen de familias con diferentes niveles socioeconómicos y cuentan con distintos recursos socioculturales (Alegre, 2010; Jaume y Gasparini, 2013).

El "efecto par" o "efecto de composición" que está detrás de estas discusiones se vincula a la influencia de los grupos en la determinación de los resultados individuales. La idea básica sugiere que, si los estudiantes de menores capacidades o recursos se concentran en determinadas escuelas o aulas, éstos tendrán menores oportunidades de lograr aprendizajes, pues la mayor parte de sus compañeros presentarán características similares (Dupriez, 2010; Durlauf, 2006). En contraposición, las escuelas integradas se convierten en comunidades de aprendizaje en las que se aprende tanto de los docentes como de los pares (García-Huidobro, 2007). Además, existe evidencia de que las escuelas menos segregadas generan mejores resultados escolares, e incluso mejores resultados de acceso y permanencia en la educación post-secundaria (Orfield y Lee, 2005), en contraposición a las escuelas segregadas, en las que los grupos más vulnerables son los más afectados (Murillo y Carrillo, 2020).

Finalmente, la segregación afecta el plano de las políticas educativas, lo cual está relacionado, a su vez, con la eficacia y equidad de los sistemas, dado que sistemas educativos segregados dificultan la posibilidad de aplicar programas exitosos de mejoramiento escolar a un nivel institucional en el largo plazo ( $\mathrm{Ru}-$ bia, 2013; Valenzuela et al., 2010).
Bajo estas reflexiones, este estudio propone aportar una imagen de la magnitud de la segregación escolar por nivel socioeconómico en República Dominicana y sus 18 regionales educativas, ${ }^{2}$ con una mirada específica en las escuelas del nivel primaria situadas en contextos urbanos.

\section{Metodología}

Para cumplir con el objetivo de este estudio se realizó una explotación especial de la base de datos de la Evaluación Diagnóstica Nacional (EDN), realizada por la Dirección de Evaluación de la Calidad del Ministerio de Educación de República Dominicana (MINERD). Esta evaluación censal fue desarrollada por primera vez en 2017 y tiene como propósito identificar las competencias que han logrado desarrollar los estudiantes en lengua española y matemática establecidas en el currículo nacional para orientar las acciones de las distintas instancias del sistema educativo hacia la mejora de la calidad y la equidad educativa (Ministerio de Educación, 2018).

La población de la EDN fueron los estudiantes del tercer grado de primaria, que es el último del primer ciclo de este nivel. Tras la necesaria limpieza de datos, se contó finalmente con 157 mil 754 estudiantes distribuidos en 7 mil 791 escuelas de todo el país y a partir de ello se seleccionaron 106 mil 526 alumnos distribuidos en 4 mil 232 escuelas ubicadas en entornos urbanos (Tabla 1). El propósito de separar las escuelas rurales de las urbanas responde a la necesidad de controlar el efecto de la segregación residencial, propia de las zonas rurales latinoamericanas, dada la alta segregación escolar que se da en lugares con una alta concentración de familias de determinado nivel socioeconómico y limitada oferta escolar.

\footnotetext{
${ }^{2}$ El Ministerio de Educación de República Dominicana cuenta con 18 direcciones regionales y 108 distritos educativos que le apoyan en su estructura organizativa.
} 
Tabla 1. Participantes y sus características

\begin{tabular}{|c|c|c|c|c|c|c|}
\hline & & Total & & & En entorno urban & \\
\hline & $\begin{array}{l}\text { Núm. } \\
\text { alumnos }\end{array}$ & Núm. escuelas & ISE promedio & $\begin{array}{l}\text { Núm. } \\
\text { alumnos }\end{array}$ & Núm. escuelas & ISE promedio \\
\hline Azua & 8,229 & 364 & -0.3852 & 4,820 & 147 & -0.2009 \\
\hline Bahoruco & 3,319 & 139 & -0.6120 & 1,689 & 46 & -0.3313 \\
\hline Barahona & 4,071 & 161 & -0.5948 & 2,744 & 67 & -0.4574 \\
\hline Cotui & 5,340 & 326 & 0.0209 & 2,855 & 119 & 0.1519 \\
\hline Higüey & 7,356 & 325 & -0.1962 & 4,322 & 130 & 0.0394 \\
\hline La Vega & 9,863 & 494 & -0.0057 & 4,044 & 122 & 0.1424 \\
\hline Mao & 3,350 & 194 & -0.2337 & 2,134 & 68 & -0.1200 \\
\hline Monte Cristi & 2,896 & 191 & -0.1964 & 1,441 & 47 & -0.0276 \\
\hline Monte Plata & 3,327 & 235 & -0.4192 & 1,340 & 38 & -0.2141 \\
\hline Nagua & 4,047 & 282 & 0.0220 & 1,684 & 69 & 0.2781 \\
\hline Puerto Plata & 5,030 & 332 & -0.1774 & 2,859 & 111 & -0.0152 \\
\hline San Cristóbal & 10,984 & 478 & -0.0628 & 6,111 & 235 & 0.0861 \\
\hline $\begin{array}{l}\text { San Francisco } \\
\text { de Macorís }\end{array}$ & 5,733 & 391 & -0.0181 & 2,919 & 110 & 0.1879 \\
\hline $\begin{array}{l}\text { San Juan de la } \\
\text { Maguana }\end{array}$ & 5,612 & 368 & -0.5321 & 2,203 & 66 & -0.2200 \\
\hline $\begin{array}{l}\text { San Pedro de } \\
\text { Macorís }\end{array}$ & 11,691 & 522 & -0.1520 & 8,700 & 302 & -0.0225 \\
\hline Santiago & 15,021 & 653 & 0.1202 & 10,358 & 387 & 0.2318 \\
\hline $\begin{array}{l}\text { Santo } \\
\text { Domingo I }\end{array}$ & 26,878 & 1,240 & 0.4343 & 22,621 & 1,108 & 0.4861 \\
\hline $\begin{array}{l}\text { Santo } \\
\text { Domingo II }\end{array}$ & 25,007 & 1,096 & 0.2385 & 23,682 & 1,060 & 0.2665 \\
\hline $\begin{array}{l}\text { Total/ } \\
\text { promedio }\end{array}$ & 157,754 & 7,791 & -0.1528 & 106,526 & 4,232 & 0.1722 \\
\hline
\end{tabular}

Fuente: elaboración propia a partir de datos de la Evaluación Diagnóstica de Tercer Grado 2017.

La variable criterio utilizada para el estudio es el índice socioeconómico (ISE), el cual fue construido por el Ministerio de Educación con base en diversas variables de tenencia de bienes en el hogar y nivel educativo de la madre (Ministerio de Educación, 2018). Esta variable se presenta tipificada.

Se utilizan los índices de Gorard para medir la dimensión de uniformidad de la segregación, y el de aislamiento para medir la dimensión de exposición. Esta doble estimación nos permite comprender en profundidad el fenómeno de la segregación escolar en el país en sus dimensiones principales, dado que ambos índices se complementan. Las magnitudes se manifiestan en un rango que va de 0 (no existe segregación) a 1 (segregación total), aunque se reconoce que la segregación escolar es un fenómeno relativo porque nunca llegará a desaparecer del todo y tampoco será absoluta.

El índice de segregación de Gorard se define como la "proporción de estudiantes desfavorecidos que tendrían que intercambiar escuelas dentro del área del análisis para que exista una distribución uniforme de este grupo entre la población" (Gorard y Smith, 2004: 19). 
Matemáticamente se expresa de la siguiente forma:

$$
G=\frac{1}{2} \sum_{i=1}^{k}\left|\frac{x_{1 i}}{X_{1}}-\frac{T_{i}}{T}\right|
$$

Donde, para país, $\mathrm{x}_{1 i}$ representa el número de alumnos del grupo minoritario en la escuela $i ; \mathrm{X}_{1}$ es el número total de estudiantes del grupo minoritario en todas las escuelas del país; $\mathrm{T}_{i}$ es el número total de alumnos en la escuela $i$; $\mathrm{y}$, corresponde al número total de alumnos en cada país.

El índice de aislamiento propuesto por Lieberson (1981) mide la "probabilidad de que un estudiante del grupo minoritario se encuentre en su escuela con otro miembro de su mismo grupo" (Murillo, 2016: 43). De esta forma, cuanto mayor sea el aislamiento del grupo, menor será su exposición a los miembros del resto de grupos. El procedimiento de estimación es:

$$
A=\sum_{i=1}^{k} \frac{x_{1 i}}{X_{1}} \frac{x_{1 i}}{T_{i}}
$$

Donde, $\mathrm{x}_{1 i}$ representa el número de alumnos del grupo minoritario en la escuela $i$; $\mathrm{X}_{1}$ es el número total de estudiantes minoritarios, respectivamente, en todas las escuelas del país; $y$ $\mathrm{T}_{i}$ es el número total de alumnos en la escuela $i$.

Para estimar ambos índices se optó por definir cuatro grupos minoritarios, cada uno de los cuales se compara con el grupo mayoritario, que sería el resto de los estudiantes. Esta distribución nos ayuda metodológicamente a identificar la ventaja relativa de un grupo sobre otro al relevar la noción de "grupos segregados”. Así tenemos el 10 y el 25 por ciento de los estudiantes con familias de menor índice socioeconómico (percentil 10, P10 y primer cuartil, Q1) y el 25 y el 10 por ciento de los estudiantes con familias de mayor índice socioeconómico (cuarto cuartil, Q4 y percentil 90, P90). También se estimó la puntuación promedio de la segregación para los cuatro colectivos.

\section{Resultados}

Un primer resultado que encontramos en este estudio es que la segregación escolar por nivel socioeconómico de las escuelas primarias de República Dominicana tiene una magnitud considerada medio-alta (mayor a 0.4 ) en sus dimensiones de uniformidad y de exposición.

Efectivamente, en la dimensión de uniformidad, el índice de Gorard (IG) es de 0.44 para el total de escuelas (Tabla 2) y de 0.42 para las escuelas sólo urbanas (Tabla 3). Estos datos indican que, en promedio, 4 de cada 10 estudiantes dominicanos de educación primaria deberían cambiarse de escuela para lograr un reparto igual a la presencia de los estudiantes de su mismo grupo en la población total.

Complementariamente, en la dimensión de exposición, la magnitud del índice de aislamiento (IA) es de 0.40 en todas las escuelas (Tabla 4) y 0.37 en las escuelas urbanas (Tabla 5), lo que indica que la probabilidad de que un estudiante del grupo minoritario se encuentre en su escuela con otro estudiante de su mismo grupo es de 40 por ciento en cualquier escuela del nivel de primaria del país. En ambos casos vemos que la tendencia de la segregación medio-alta es muy parecida, tanto para el total de escuelas como para las escuelas sólo urbanas.

Un segundo resultado nos indica que esta distribución no es la misma si se identifican los grupos minoritarios (Gráfica 1) en ambas dimensiones. Un primer aspecto que destaca a simple vista es que la segregación, en su dimensión de uniformidad (índice de Gorard), es mayor conforme el grupo minoritario es más pequeño (P10 y P90), mientras que, para la dimensión de exposición (índice de aislamiento), la segregación es más alta en los cuartiles (Q1 y Q4). Esto es previsible dadas las características de este último índice: la probabilidad de encontrarse con alguien de su mismo grupo depende de la cantidad de personas de ese grupo (Murillo, 2016). 
Sin embargo, es importante destacar las tendencias: la segregación es claramente superior para los estudiantes con familias de mayor nivel socioeconómico (IG de 0.60 en el P90 e IA de 0.52 en el Q4), seguido de los estudiantes con familias con menor nivel socioeconómico ( 0.42 en el P10 del IG y en el Q1 del IA).
Estos datos indican que la segregación escolar en República Dominicana se da en mayor medida entre los estudiantes de familias con mayor nivel socioeconómico, y que la segregación es menor hacia los colectivos más desfavorecidos. Para escuelas sólo urbanas, las tendencias son exactamente iguales.

Gráfica 1. Segregación escolar por nivel socioeconómico en República Dominicana según el grupo minoritario considerado

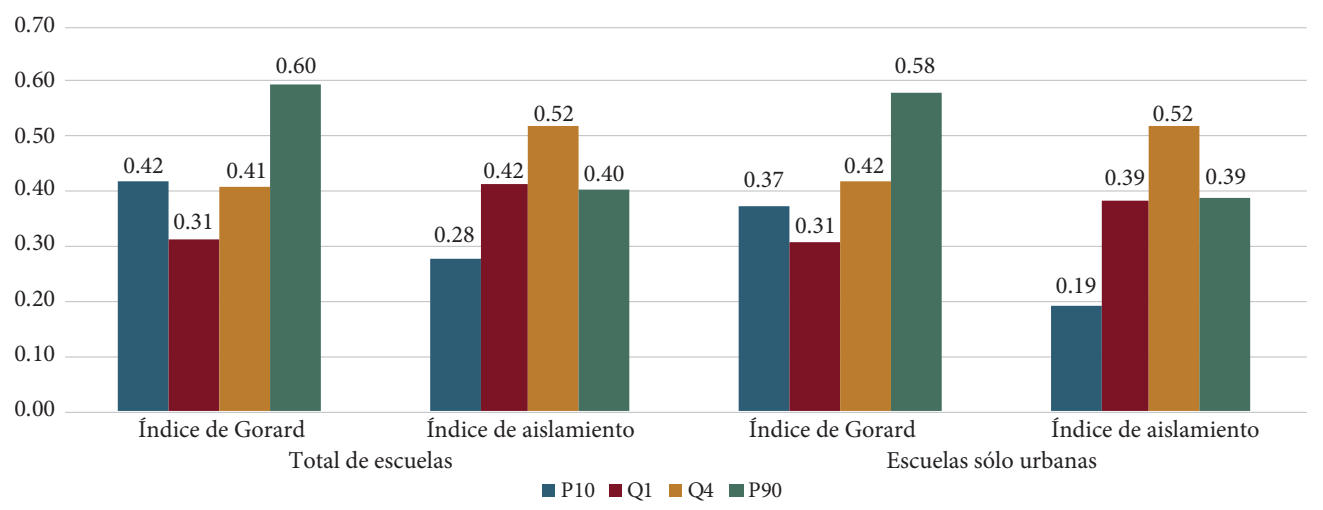

* Se considera para el total de escuelas y para escuelas urbanas. Índices de Gorard y de aislamiento para el 10 y 25 por ciento de los estudiantes con familias de menor índice socioeconómico (P10 y Q1) y el 25 y 10 por ciento de los estudiantes con familias de mayor índice socioeconómico (Q4 y P90).

Fuente: elaboración propia a partir de datos de la Evaluación Diagnóstica de Tercer Grado 2017.

Un tercer resultado evidencia que hay fuertes diferencias en función de la regional educativa (Gráfica 2): al considerar el promedio de la segregación con los cuatro colectivos considerados, se puede afirmar que Santo Domingo II y San Juan de la Maguana son las regionales que presentan una mayor segregación escolar por nivel socioeconómico de toda República Dominicana (índice G de 0.52 para ambos). Por el contrario, Cotui (0.34) y Mao (0.36) son las que presentan una menor segregación promedio.

\section{Gráfica 2. Segregación escolar por nivel socioeconómico en}

República Dominicana y sus regionales educativas. Índice de Gorard promedio

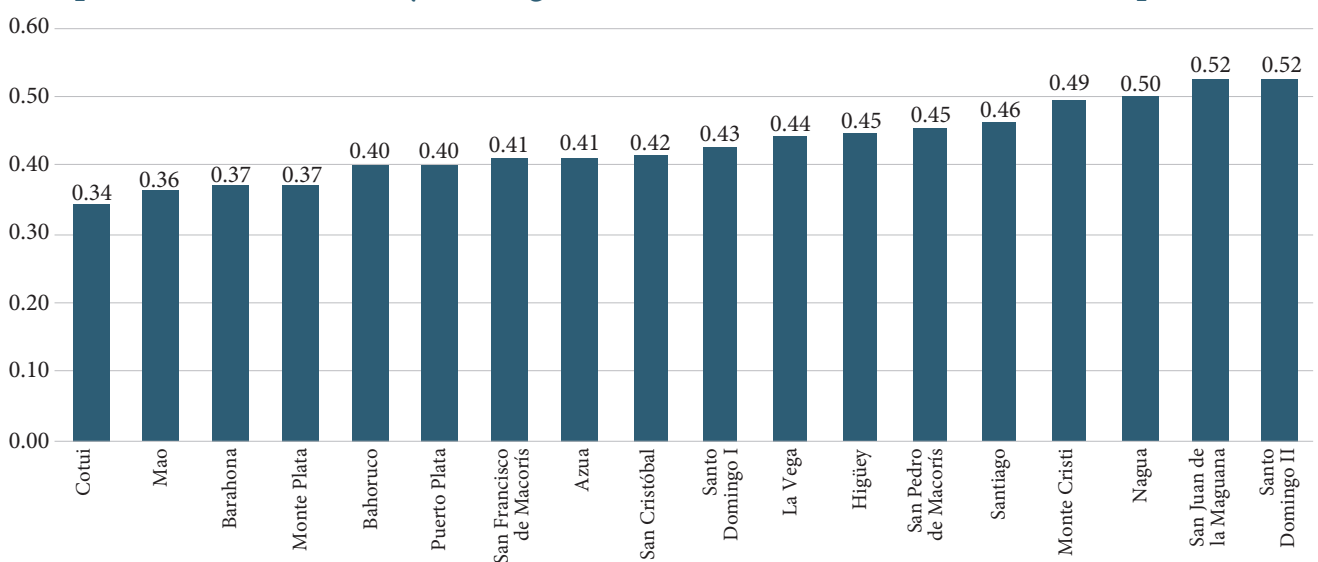

Fuente: elaboración propia a partir de datos de la Evaluación Diagnóstica de Tercer Grado 2017. 
Las cifras anteriores pueden ser matizadas si se considera la segregación para cada colectivo (Tabla 2). Efectivamente:

- Para los estudiantes con menor nivel socioeconómico, la regional educativa con segregación más alta en el percentil 10 (P10) y en el primer cuartil (Q1) es San Juan de la Maguana ( 0.56 y 0.39 respectivamente), mientras que Cotui es la que menos segregación tiene en ambos grupos (0.31 y 0.21 respectivamente).

- Para el grupo de estudiantes con mayor nivel socioeconómico, las regionales educativas con la segregación más alta en el último cuartil (Q4) y en el percentil 90 (P90) son Santo Domingo II (0.50 y $0.65)$ y Nagua $(0.44$ y 0.64$)$. En el extremo opuesto se encuentran Mao ( 0.26 y 0.43 ) y Cotui (0.28 y 0.43).

Tabla 2. Segregación escolar por nivel socioeconómico en República Dominicana y sus regionales educativas

Índice de Gorard para P10, Q1, Q4, P90 como grupos minoritarios, y promedio

\begin{tabular}{|c|c|c|c|c|c|}
\hline & P10 & Q1 & Q4 & P90 & Promedio \\
\hline República Dominicana & 0.4205 & 0.3122 & 0.4109 & 0.5951 & 0.4347 \\
\hline Azua & 0.3950 & 0.2820 & 0.3252 & 0.4858 & 0.4137 \\
\hline Bahoruco & 0.3713 & 0.2911 & 0.3216 & 0.4615 & 0.4031 \\
\hline Barahona & 0.4000 & 0.2762 & 0.3070 & 0.4585 & 0.3700 \\
\hline Cotui & 0.3116 & 0.2088 & 0.2818 & 0.4309 & 0.3424 \\
\hline Higüey & 0.4511 & 0.3159 & 0.3441 & 0.4929 & 0.4461 \\
\hline La Vega & 0.3768 & 0.2876 & 0.3657 & 0.5582 & 0.4418 \\
\hline Mao & 0.3675 & 0.2501 & 0.2631 & 0.4278 & 0.3634 \\
\hline Monte Cristi & 0.5100 & 0.3146 & 0.3705 & 0.5850 & 0.4936 \\
\hline Monte Plata & 0.3722 & 0.2463 & 0.2772 & 0.4468 & 0.3728 \\
\hline Nagua & 0.4082 & 0.3005 & 0.4347 & 0.6426 & 0.4967 \\
\hline Puerto Plata & 0.4071 & 0.2766 & 0.3100 & 0.4592 & 0.4040 \\
\hline San Cristóbal & 0.3436 & 0.2631 & 0.3680 & 0.5178 & 0.4157 \\
\hline San Francisco de Macorís & 0.3609 & 0.2492 & 0.3471 & 0.5258 & 0.4121 \\
\hline San Juan de la Maguana & 0.5562 & 0.3917 & 0.3690 & 0.5411 & 0.5164 \\
\hline San Pedro de Macorís & 0.3795 & 0.2905 & 0.3862 & 0.5761 & 0.4542 \\
\hline Santiago & 0.3614 & 0.2790 & 0.4035 & 0.6163 & 0.4618 \\
\hline Santo Domingo I & 0.3647 & 0.3102 & 0.3553 & 0.4996 & 0.4265 \\
\hline Santo Domingo II & 0.3980 & 0.3400 & 0.4946 & 0.6485 & 0.5248 \\
\hline
\end{tabular}

Fuente: elaboración propia a partir de datos de la Evaluación Diagnóstica de Tercer Grado 2017.

En todo caso llaman la atención algunos detalles que se desprenden del análisis anterior. Un primer detalle es que en todas las regionales educativas, con una excepción, la mayor segregación se da en el 10 por ciento de los alumnos con familias de mayor nivel socioeconómico (P90). La excepción es San Juan de la Maguana, donde es mayor la segregación para el 10 por ciento de los estudiantes con menor nivel socioeconómico (P10).

El otro detalle es que también, en todas las regionales educativas y con la misma excepción, la menor segregación se da con 25 por ciento de los estudiantes con familias de 
menor ISE (Q1). En San Juan de la Maguana la menor segregación se da con el 25 por ciento de los estudiantes con mayor ISE (Q4).

Se identifica, además, que la diferencia de la segregación para cada colectivo es muy variable: mientras que en Nagua y Santiago se da la mayor diferencia (de 0.64 para el P90 y de 0.30 con el Q1 en Nagua; y de 0.62 (P90) y 0.28 (Q1) en Santiago), en San Juan de la Maguana la diferencia de ambas es de 0.15 y en Bahoruco de 0.17.
Especialmente interesante es la comparación de la segregación escolar del conjunto de las escuelas y de las escuelas en contextos urbanos (Gráfica 3). Se visibiliza perfectamente que, aunque en todas las regionales educativas la segregación es inferior cuando se quitan las escuelas situadas en zonas rurales, la diferencia entre ambos datos es muy diferente en cada regional. Efectivamente, esa diferencia es muy grande en San Juan de la Maguana (0.22), algo menor en Bahoruco (0.13) y muy pequeña en Santiago y La Vega (con 0.02 de diferencia).

Gráfica 3. Segregación escolar por nivel socioeconómico en las regionales educativas en el total de escuelas y en las escuelas en contextos urbanos Índice de Gorard promedio

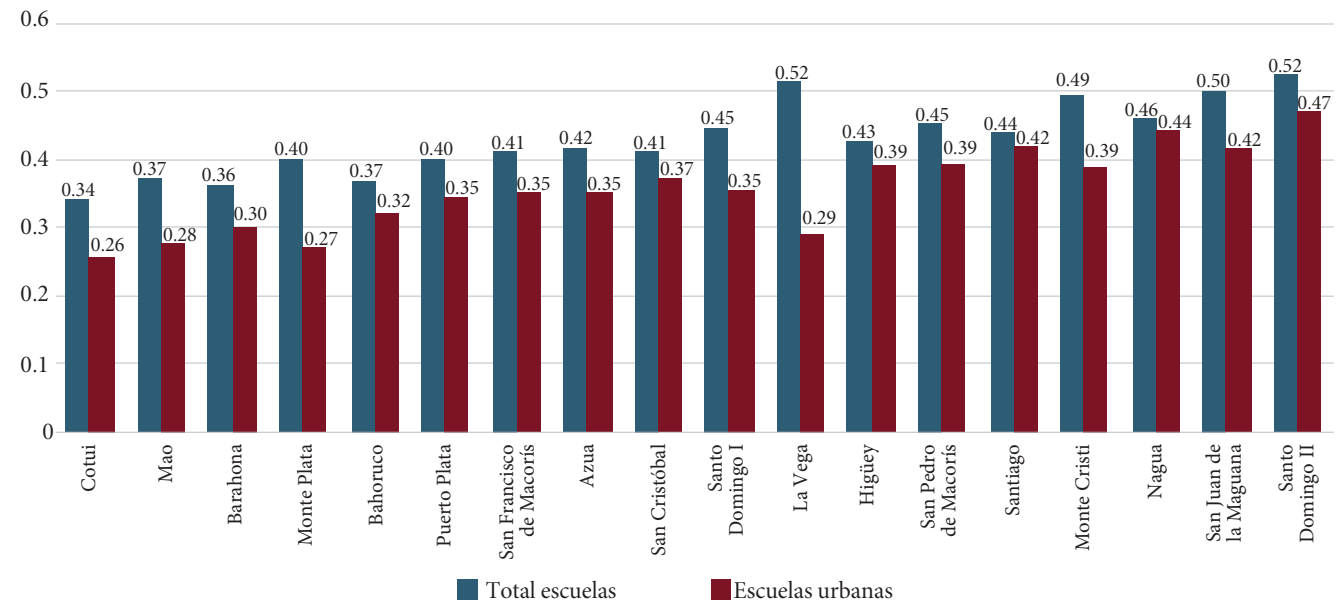

Fuente: elaboración propia a partir de datos de la Evaluación Diagnóstica de Tercer Grado 2017.

La mirada de la segregación en escuelas urbanas en cada regional educativa, para cada de uno de los cuatro grupos minoritarios considerados (Tabla 3), aporta la siguiente imagen:

- Para los estudiantes con familias de menor nivel socioeconómico, Santo Domingo II es la regional educativa con mayor segregación en las escuelas situadas en contextos urbanos, tanto en el percentil 10 (0.40), como en el primer cuartil (0.34). En el otro extremo, presentan menor segregación Cotui (0.27 y 0.19 respectivamente) y Monte Plata (0.26 y 0.18).
- Para los estudiantes con familias de mayor nivel socioeconómico, las regionales educativas con mayor segregación, en el último cuartil (Q4) y en el percentil 90, son Santo Domingo II (0.50 y 0.65) y Santiago (0.44 y 0.64). En el lado contrario, las que menos segregación presentan son Cotui (0.22 y 0.34) y Bahoruco (0.24 y 0.26).

Así, desde una mirada global (que incluye el total de escuelas) y desde la dimensión de uniformidad, las escuelas más segregadas se presentan en San Juan de la Maguana, para los grupos de menor nivel socioeconómico, 
Tabla 3. Segregación escolar por nivel socioeconómico en República Dominicana y sus regiones para escuelas urbanas Índice de Gorard para P10, Q1, Q4, P90 como grupos minoritarios, y promedio

\begin{tabular}{|c|c|c|c|c|c|}
\hline & P10 & Q1 & Q4 & P90 & Promedio \\
\hline República Dominicana & 0.3736 & 0.3075 & 0.4190 & 0.5815 & 0.4204 \\
\hline Azua & 0.3181 & 0.2536 & 0.3362 & 0.4793 & 0.3468 \\
\hline Bahoruco & 0.2682 & 0.2150 & 0.2420 & 0.3599 & 0.2713 \\
\hline Barahona & 0.3085 & 0.2307 & 0.2915 & 0.4571 & 0.3220 \\
\hline Cotui & 0.2731 & 0.1882 & 0.2175 & 0.3442 & 0.2558 \\
\hline Higüey & 0.3409 & 0.2609 & 0.3469 & 0.4703 & 0.3548 \\
\hline La Vega & 0.3791 & 0.3240 & 0.4132 & 0.5573 & 0.4184 \\
\hline Mao & 0.2948 & 0.1919 & 0.2764 & 0.4296 & 0.2982 \\
\hline Monte Cristi & 0.3625 & 0.2523 & 0.3412 & 0.5995 & 0.3889 \\
\hline Monte Plata & 0.2642 & 0.1755 & 0.2478 & 0.4187 & 0.2765 \\
\hline Nagua & 0.3955 & 0.2886 & 0.4139 & 0.5634 & 0.4153 \\
\hline Puerto Plata & 0.3082 & 0.2680 & 0.3256 & 0.4858 & 0.3469 \\
\hline San Cristóbal & 0.3137 & 0.2758 & 0.3175 & 0.5010 & 0.3520 \\
\hline San Francisco de Macorís & 0.2974 & 0.2539 & 0.3936 & 0.5458 & 0.3727 \\
\hline San Juan de la Maguana & 0.2761 & 0.2204 & 0.2814 & 0.3900 & 0.2920 \\
\hline San Pedro de Macorís & 0.3213 & 0.2846 & 0.3907 & 0.5723 & 0.3922 \\
\hline Santiago & 0.3808 & 0.3085 & 0.4434 & 0.6438 & 0.4441 \\
\hline Santo Domingo I & 0.3761 & 0.3138 & 0.3579 & 0.5020 & 0.3874 \\
\hline Santo Domingo II & 0.3965 & 0.3424 & 0.4954 & 0.6472 & 0.4704 \\
\hline
\end{tabular}

Fuente: elaboración propia a partir de datos de la Evaluación Diagnóstica de Tercer Grado 2017.

y Santo Domingo II y Nagua para los grupos más adinerados. Por otro lado, si sólo vemos las escuelas urbanas en su dimensión de uniformidad, Santo Domingo II sigue siendo la regional más segregada para los cuatro grupos minoritarios. Cotui es la regional que menos segregación tiene, tanto para el total de escuelas como sólo para las urbanas y en los cuatro grupos minoritarios.

Sobre la dimensión de exposición, las diferencias entre las regionales educativas según grupos minoritarios para el total de escuelas (Tabla 4) confirman lo encontrado en los análisis previos:

- Al considerar los grupos de estudiantes con menor nivel socioeconómico (P10 y Q1), las regionales educativas con mayor segregación son San Juan de la Maguana (0.40 y 0.51) y Monte Cristi (0.37 y 0.44). En el otro extremo, Cotui es la regional con menor segregación en ambos grupos ( 0.18 y 0.34 ).

- Para los grupos de estudiantes con mayor nivel socioeconómico (Q4 y P90), las regionales con mayor segregación son Santo Domingo II (0.61 y 0.44) y Santiago (0.53 y 0.46), mientras que Monte Plata (0.37 y 0.23$)$ tiene la menor segregación, en ambos grupos.

En la misma línea que el análisis previo se encuentra que la dimensión de exposición de la segregación escolar para escuelas urbanas (0.37) es más baja que la encontrada para el total de escuelas (0.40). El análisis detallado por 
Tabla 4. Segregación escolar por nivel socioeconómico

en República Dominicana y sus regionales educativas

Índice de aislamiento para P10, Q1, Q4, P90 como grupos minoritarios, y promedio

\begin{tabular}{|c|c|c|c|c|c|}
\hline & P10 & Q1 & Q4 & P90 & Promedio \\
\hline República Dominicana & 0.2758 & 0.4154 & 0.5205 & 0.4022 & 0.4035 \\
\hline Azua & 0.2500 & 0.3937 & 0.4457 & 0.3338 & 0.3558 \\
\hline Bahoruco & 0.2637 & 0.4023 & 0.3992 & 0.2326 & 0.3245 \\
\hline Barahona & 0.2984 & 0.4100 & 0.4183 & 0.3204 & 0.3618 \\
\hline Cotui & 0.1782 & 0.3362 & 0.3940 & 0.2452 & 0.2884 \\
\hline Higüey & 0.3039 & 0.4291 & 0.4632 & 0.3313 & 0.3819 \\
\hline La Vega & 0.2173 & 0.3884 & 0.4746 & 0.3703 & 0.3626 \\
\hline Mao & 0.2288 & 0.3659 & 0.3829 & 0.2595 & 0.3093 \\
\hline Monte Cristi & 0.3648 & 0.4378 & 0.4591 & 0.4138 & 0.4189 \\
\hline Monte Plata & 0.2224 & 0.3705 & 0.3681 & 0.2339 & 0.2987 \\
\hline Nagua & 0.2388 & 0.3995 & 0.5111 & 0.3780 & 0.3819 \\
\hline Puerto Plata & 0.2615 & 0.3894 & 0.4270 & 0.3098 & 0.3469 \\
\hline San Cristóbal & 0.2094 & 0.3669 & 0.4637 & 0.3245 & 0.3411 \\
\hline San Francisco de Macorís & 0.2199 & 0.3632 & 0.4778 & 0.3657 & 0.3566 \\
\hline San Juan de la Maguana & 0.3983 & 0.5055 & 0.4667 & 0.3687 & 0.4348 \\
\hline San Pedro de Macorís & 0.2390 & 0.3992 & 0.4868 & 0.3565 & 0.3704 \\
\hline Santiago & 0.1907 & 0.3678 & 0.5278 & 0.4572 & 0.3859 \\
\hline Santo Domingo I & 0.1877 & 0.3863 & 0.4474 & 0.2974 & 0.3297 \\
\hline Santo Domingo II & 0.1953 & 0.4035 & 0.6138 & 0.4358 & 0.4121 \\
\hline
\end{tabular}

Fuente: elaboración propia a partir de datos de la Evaluación Diagnóstica de Tercer Grado 2017.

regionales educativas (Tabla 5), nos indica una variación de entre 0.41 y 0.25 .

- Al considerar como grupos minoritarios los estudiantes con menor ISE, encontramos que la regional educativa con mayor segregación en escuelas urbanas es Santo Domingo II ( 0.20 y 0.40). En el otro extremo, Cotui (0.14 y 0.31) y Monte de Plata (0.14 y 0.31) son las que presentan una menor segregación.

- Para los grupos minoritarios con mayor nivel socioeconómico (Q4 y P90), Santo Domingo II (0.61 y 0.44) y Santiago (0.56 y 0.40) presentan la mayor segregación, mientras que Cotui $(0.35 \mathrm{y}$ 0.17 ) y Bahoruco ( 0.35 y 0.19 ) son las que tienen menos segregación.
Una mirada global a la dimensión de exposición nos muestra que la situación es muy similar a la encontrada en la dimensión de uniformidad: Santo Domingo II y Santiago son las regionales más segregadas en los grupos minoritarios de mayor nivel socioeconómico, mientras que Cotui es la menos segregada en los cuatro grupos, tanto para el total de escuelas como sólo para las urbanas.

Un interesante análisis es relacionar el nivel socioeconómico de las familias de los estudiantes de cada regional educativa con la segregación escolar para las escuelas urbanas. Al estimar el índice Rho de Spearman se encontró que hay una correlación significativa y alta entre el índice socioeconómico de cada regional y la segregación en las escuelas urbanas. Concretamente un índice Rho de 0.67 
Tabla 5. Segregación escolar por nivel socioeconómico

en República Dominicana y sus regionales educativas para escuelas urbanas

Índice de aislamiento para P10, Q1, Q4, P90 como grupos minoritarios, y promedio

\begin{tabular}{|c|c|c|c|c|c|}
\hline & $\mathrm{P} 10$ & Q1 & Q4 & P90 & Promedio \\
\hline Rep. Dominicana & 0.1926 & 0.3857 & 0.5197 & 0.3866 & 0.3712 \\
\hline Azua & 0.1664 & 0.3498 & 0.4471 & 0.3250 & 0.3221 \\
\hline Bahoruco & 0.1485 & 0.3200 & 0.3464 & 0.1939 & 0.2522 \\
\hline Barahona & 0.1542 & 0.3269 & 0.4039 & 0.3406 & 0.3064 \\
\hline Cotui & 0.1446 & 0.3100 & 0.3501 & 0.1734 & 0.2445 \\
\hline Higüey & 0.1807 & 0.3597 & 0.4605 & 0.3137 & 0.3286 \\
\hline La Vega & 0.1879 & 0.4048 & 0.5154 & 0.3377 & 0.3614 \\
\hline Mao & 0.1584 & 0.3163 & 0.3828 & 0.2419 & 0.2749 \\
\hline Monte Cristi & 0.1936 & 0.3600 & 0.4591 & 0.4541 & 0.3667 \\
\hline Monte Plata & 0.1472 & 0.3132 & 0.3560 & 0.2069 & 0.2558 \\
\hline Nagua & 0.2039 & 0.3607 & 0.4771 & 0.2876 & 0.3323 \\
\hline Puerto Plata & 0.1586 & 0.3567 & 0.4373 & 0.3121 & 0.3162 \\
\hline San Cristóbal & 0.1675 & 0.3645 & 0.5001 & 0.3151 & 0.3368 \\
\hline San Francisco de Macorís & 0.1602 & 0.3456 & 0.4959 & 0.3553 & 0.3392 \\
\hline San Juan de la Maguana & 0.1539 & 0.3330 & 0.3802 & 0.2184 & 0.2714 \\
\hline San Pedro de Macorís & 0.1818 & 0.3675 & 0.4827 & 0.3532 & 0.3463 \\
\hline Santiago & 0.1828 & 0.3964 & 0.5598 & 0.4428 & 0.3954 \\
\hline Santo Domingo I & 0.1894 & 0.3901 & 0.4488 & 0.3012 & 0.3324 \\
\hline Santo Domingo II & 0.2010 & 0.4033 & 0.6137 & 0.4384 & 0.4141 \\
\hline
\end{tabular}

Fuente: elaboración propia a partir de datos de la Evaluación Diagnóstica de Tercer Grado 2017.

Gráfica 4. Relación entre el ISE y la segregación escolar por nivel socioeconómico de las escuelas urbanas en cada regional educativa

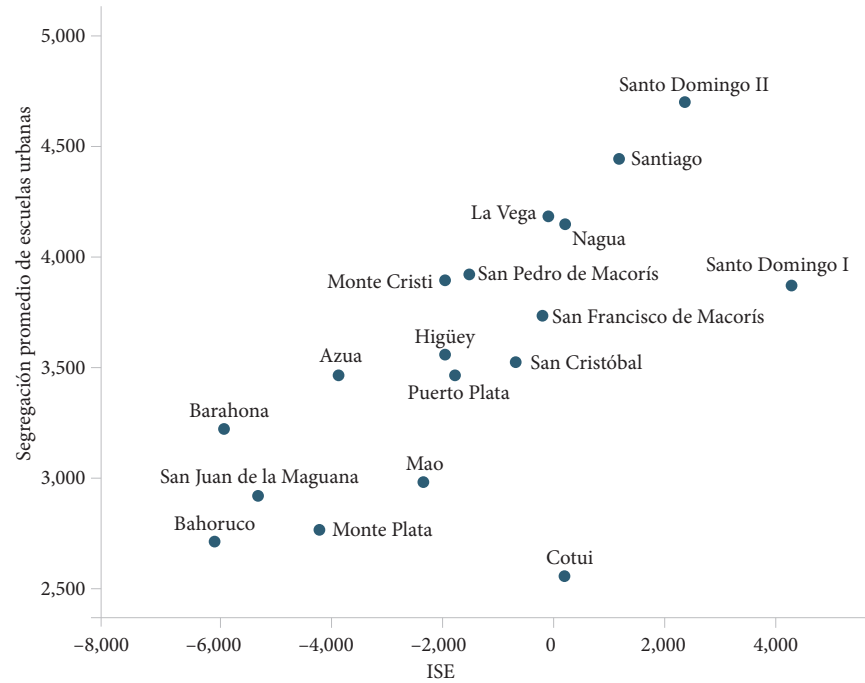

Fuente: elaboración propia a partir de datos de la Evaluación Diagnóstica de Tercer Grado 2017. 
para el P10, de 0.74 para el Q1, de 0.69 con el Q4 y de 0.63 con el P90. En la Gráfica 4 se observa la relación entre el nivel socioeconómico (ISE) de cada regional educativa y le segregación escolar promedio en escuelas urbanas.

La alta relación entre el nivel socioeconómico de la regional educativa y la segregación promedio de las escuelas urbanas es uno de los datos más llamativos de esta investigación, ya que éstos apuntan a que cuanto mayor nivel socioeconómico tiene una regional, mayor es su segregación escolar; esta segregación, como hemos visto, se da especialmente en los grupos de estudiantes de familias más adineradas. De esta manera, regionales como Santo Domingo II y Santiago tienen un mayor ISE y mayor segregación, mientras que regionales como Cotui o Bahoruco tienen menor ISE y menos segregación. Esta información nos da indicios para pensar en términos de causaefecto; sin embargo, es necesario profundizar mucho más en este sentido.

\section{Conclusiones}

Este estudio ha encontrado, como primer hallazgo, que la magnitud de la segregación escolar en las escuelas de primaria de República Dominicana es medio-alta, tanto para el total de escuelas (sobre el 0.4 en ambos índices) como para las escuelas urbanas (IG de 0.42 e IA de 0.37).

Las cifras encontradas para el conjunto de las escuelas de primaria de República Dominicana son análogas a las obtenidas por Murillo (2016) y Murillo et al. (2020), lo que da robustez a los resultados. En ambos estudios, con datos del TERCE y utilizando el índice de Gorard, se encontró una segregación de 0.42 para el 10 por ciento de los estudiantes de familias con menor nivel socioeconómico (P10), al igual que los 0.42 hallados en este trabajo; de 0.30 para Q1, frente a 0.31; y de 0.36 para el Q4, frente a los 0.41. En el caso del índice de aislamiento, en ambos estudios se encontró una segregación de 0.26 para el P10 frente a
0.27 encontrados en este estudio; de 0.39 para Q1, frente a 0.41 ; y de 0.46 para Q4. La casi coincidencia de los datos encontrados indica una coherencia con los resultados de este trabajo. Las diferencias pueden deberse a la distinta variable criterio utilizada (en un caso es el índice socioeconómico, en el otro es el índice socioeconómico y cultural), así como la fecha de cada evaluación (2013 y 2017). Además, este estudio usa datos censales, mientras que los datos del TERCE son muestrales.

Un segundo hallazgo importante que caracteriza al sistema educativo dominicano es que la segregación, en sus dos dimensiones, es mayor en los grupos de mayor nivel socioeconómico. Así, la magnitud de la segregación escolar en los grupos más ricos es muy alta, tanto para el total de escuelas (índice $\mathrm{G}$ de 0.60 para el P90 e índice A de 0.52 para el Q4), como para las escuelas sólo urbanas (índice $\mathrm{G}$ de 0.58 para el P90 e índice A de 0.52 para el Q4).

La aportación de este trabajo es brindar datos de la magnitud de la segregación escolar en las diferentes regionales educativas, a partir de los cuales se verifican situaciones dispares. En ese sentido, un tercer hallazgo tiene que ver con las diferencias en las magnitudes de la segregación entre las regionales, así como al interior de éstas en cuanto a la segregación en el total de sus escuelas y las escuelas urbanas. Esta información se complementa con un último hallazgo relacionado a la estrecha relación encontrada entre el nivel socioeconómico de las regionales educativas y la segregación escolar promedio en sus escuelas urbanas.

La segregación residencial podría explicar en parte estos resultados. Es decir, una mayor segregación en regionales como Santo Domingo II y Santiago, y una menor segregación en regionales como Cotui y Bahoruco, podría deberse a la organización misma de los territorios en cuanto a la distribución de las zonas urbanas y rurales, así como la distribución de las familias en las ciudades. Esto último debido, principalmente, a la segmentación de barrios o distritos según los niveles socioeco- 
nómicos de sus habitantes, lo que les da o no acceso a una serie de servicios y comodidades.

Por otro lado, es importante también profundizar en el peso de la educación privada en el sistema educativo dominicano. Estudios comparados muestran que República Dominicana cuenta con un sistema público-privado menos segregador que los otros países de la región (Murillo et al., 2020), aunque tiene la mayor segregación en sus escuelas privadas (Murillo y Martínez-Garrido, 2017). Esto se debe, en parte, a los procesos de privatización que definen una oferta de alianzas públicoprivadas y escuelas de bajo costo (Verger et al., 2017) que, sin duda, tienen un rol preponderante en los niveles de segregación dentro de cada una de las regionales educativas.

Con esta información se pueden plantear más hipótesis que permitan comprender la segregación escolar en República Domicana. Aun así, es importante reconocer que el fenómeno es bastante complejo dada la interacción entre las diferentes dimensiones que generan desigualdades en la escolarización, algunas de las cuales pueden ser externas a los sistemas educativos (Bonal y Bellei, 2018). En ese sentido, la segregación geográfica o residencial, que afecta a amplios sectores de población, o los efectos de los procesos de privatización, evidencian que la segregación escolar no debe ser vista de manera aislada, como un fenómeno que atañe sólo a educación, sino que debe ser comprendido en múltiples frentes (Murillo et al., 2018).

Esta investigación es una primera aproximación descriptiva de la segregación escolar en República Dominicana y sus regionales educativas. Su gran fortaleza es contar con datos censales para aportar a la discusión educativa. En este sentido, queremos reconocer y agradecer el trabajo de la Dirección de Evaluación de la Calidad del Ministerio de Educación de República Dominicana para desarrollar la evaluación y poner a disposición de la comunidad investigadora las bases de datos que ayudan a conocer mejor el sistema educativo dominicano. También es importante reconocer que este primer estudio tiene como debilidad que apenas hace un diagnóstico superficial del tema abordado. Así, como debe ser, deja más interrogantes que respuestas: ¿qué genera esta segregación?, ¿qué consecuencias tiene para el rendimiento de los estudiantes y para la conformación de la sociedad?, ¿qué medidas habría que tomar para limitar la segregación?, entre muchas otras.

La segregación escolar es un desafío importante para República Dominicana, ya que no solamente afecta a los estudiantes y sus familias, sino también a la eficacia y la equidad del sistema educativo. Está en juego no sólo garantizar una real igualdad de oportunidades en todos los estudiantes del país, sino también contribuir a una sociedad más justa, equitativa e inclusiva. Los resultados de República Dominicana apuntan a que la segregación escolar de los estudiantes de familias más adineradas, especialmente en las regionales educativas de mayor nivel socioeconómico, es alta. Urge, por ello, implementar medidas de política educativa diferenciadas, según los distintos contextos, que trabajen por un sistema educativo menos segregado y más inclusivo, para que en el mediano y largo plazo se construya una sociedad con características análogas.

escolar público-privada en América Latina, Santiago de Chile, Naciones Unidas.

Bellei, Cristián (2013), "El estudio de la segregación económica y académica de la educación chilena”, Estudios Pedagógicos, vol. 39, núm. 1, pp. 325-345. DOI: https://doi.org/10.4067/S071807052013000100019
Arcidiácono, Malena, Guillermo Cruces, Leonardo Gasparini, David Jaume, Monserrat Serio y
Emmanuel Vázquez (2014), La segregación gación escolar y desigualdad educativa: una trilogía con final abierto”, Educação \& Sociedade, vol. 31, núm. 113, pp. 1157-1178. DOI: https:// doi.org/10.1590/S0101-73302010000400006 
Bonal, Xavier y Cristián Bellei (eds.) (2018), Understanding School Segregation. Patterns, causes and consequences of spatial inequalities in education, Londres, Bloomsbury Academic.

CARrillo, Sandra (2020), "La segregación escolar en América Latina. ¿Qué se estudia y cómo se investiga?", REICE. Revista Iberoamericana sobre Calidad, Eficacia y Cambio en Educación, vol. 18, núm. 4, pp. 345-362. DOI: https:// doi.org/10.15366/reice2020.18.4.014

Checo, Francisco (2019), Informe Luz: Balance de la implementación del ODS4 en la República Dominicana. Resumen, Santo Domingo, Foro Socioeducativo.

Coleman, James S. (1966), Equality of Educational Opportunity, Washington D.C., US Department of Health, Education and Welfare.

Comisión Económica para América Latina y el Caribe (CEPAL) (2016), La matriz de la desigualdad social en América Latina, Santiago de Chile, Naciones Unidas.

Dupriez, Vincent (2010), Methods of Grouping Learners at School, París, UNESCO.

Durlauf, Steven N. (2006), "Groups, Social Influences, and Inequality: A memberships theory perspective on poverty traps", en Samuel Bowles, Steven N. Durlauf y Karla Hoff (eds.), Poverty Traps, Princeton, Princeton University Press, pp. 141-175.

García-Huidobro, Juan Eduardo (2007), “Desigualdad educativa y segmentación del sistema escolar. Consideraciones a partir del caso chileno", Revista Pensamiento Educativo, vol. 40, núm. 1, pp. 65-85.

Gasparini, Leonardo, David Jaume, Monserrat Serio y Emmanuel Vázquez (2011), La segregación escolar en Argentina. Reconstruyendo la evidencia, Buenos Aires, CEDLAS.

Gentili, Pablo (2009), "Marchas y contramarchas. El derecho a la educación y las dinámicas de exclusión incluyente en América Latina (a sesenta años de la Declaración Universal de los Derechos Humanos)", Revista Iberoamericana de Educación, núm. 49, pp. 19-57. DOI: https://doi.org/10.35362/rie490673

Gobierno de República Dominicana-Ministerio de Educación-Dirección de Evaluación de la Calidad de la Educación (2018), Resultados de la evaluación diagnóstica del tercer grado de primaria. Informe nacional, Santo Domingo, MINERD.

Gorard, Stephen y Emma Smith (2004), “An International Comparison of Equity in Education Systems", Comparative Education, vol. 40, núm. 1, pp. 15-28. DOI: https://doi.org/10.1080 /0305006042000184863

GorARD, Stephen y Chris Taylor (2002), "What is Segregation? A comparison of measures in terms of 'strong' and 'weak' compositional invariance”, Sociology, vol. 36, núm. 4, pp. 875-895. DOI: https://doi.org/10.1177/003803850203600405
Jaume, David y Leonardo Gasparini (2013), Un estudio sobre el incremento de la segregación escolar en Argentina, Buenos Aires, CEDLAS.

Karsten, Sjoerd (2010), "School Segregation", en OECD (ed.), Equal Opportunities? The labour market integration on the children of immigrants, París, OECD Publishing, pp. 193-209.

Lieberson, Stanley (1981), "An Asymmetrical Approach to Segregation", en Cery Peach, Vaughan Robinson y Susan Smith (ed.), Ethnic Segregation in Cities, Londres, CroomHelm, pp. 61-82.

Murillo, F. Javier (2016), “Midiendo la segregación escolar en América Latina. Un análisis metodológico utilizando el TERCE”, REICE. Revista Iberoamericana sobre Calidad, Eficacia y Cambio en Educación, vol. 14, núm. 4, pp. 33-60. DOI: https://doi.org/10.15366/reice 2016.14.4.002

Murillo, F. Javier y Sandra Carrillo (2021), "Incidencia de la segregación escolar por nivel socioeconómico en el rendimiento académico. Un estudio desde Perú", Archivos Analíticos de Políticas Educativas, vol. 29, núm. 49, pp. 1-24. DOI: https://doi.org/10.14507/epaa.29.5129

Murillo, F. Javier y Cynthia Martínez-Garrido (2017), "Segregación social en las escuelas públicas y privadas en América Latina”, Educação \& Sociedade, vol. 38, núm. 140, pp. 727-750. DOI: https://doi.org/10.1590/es0101-73302017167714

Murillo, F. Javier y Cynthia Martínez-Garrido (2019), "Una mirada a la investigación educativa en América Latina a partir de sus artículos", REICE. Revista Iberoamericana sobre Calidad, Eficacia y Cambio en Educación, vol. 17, núm. 2, pp. 5-24. DOI: https://doi.org/10.15366/ reice2019.17.2.001

Murillo, F. Javier, Cynthia Duk y Cynthia Martínez-Garrido (2018), "Evolución de la segregación socioeconómica de las escuelas de América Latina", Estudios Pedagógicos, vol. 44, núm. 1, pp. 157-179. DOI: https://doi. org/10.4067/S0718-07052018000100157

Murillo, F. Javier, Cynthia Martínez-Garrido y Guillermina Belavi (2017), "Segregación escolar por origen nacional en España”, OBETS. Revista de Ciencias Sociales, vol. 12, núm. 2, pp. 395-432. DOI: https://doi.org/10.14198/OBE TS2017.12.2.04

Murillo, F. Javier, Cynthia Martínez-Garrido y Raquel Graña (2020), "Escuelas públicas para pobres, escuelas privadas para ricos: relación entre educación privada y segregación escolar de carácter socioeconómico en América Latina", Revista Científica RUNAE, núm. 5, pp. 11-22.

Orfield, Gary (2001), Schools More Separate. Consequences of a decade of resegregation, Cambridge, Harvard University Press. 
Orfield, Gary y Chungmei Lee (2005), Why Segregation Matters: Poverty and educational inequality, Cambridge, Harvard University Press.

Organisation for Economic Co-operation and Development (OECD) (2019), Balancing School Choice and Equity. An international perspective based on Pisa, París, OECD.

Pereyra, Ana (2008), "La fragmentación de la oferta educativa en América Latina: la educación pública vs. la educación privada", Perfiles Educativos, vol. 30, núm. 120, pp. 132-146.

Plá, Sebastián (2015), "Debates contemporáneos sobre la inequidad y la exclusión educativa en América Latina”, Sinéctica, núm. 44, pp. 1-19.

Reardon, Sean F. y Ann Owens (2014), "60 Years after Brown: Trends and consequences of school segregation", Annual Review of Sociology, vol. 40, pp. 199-218. DOI: https://doi. org/10.1146/annurev-soc-071913-043152
Rubia, Fernando Andrés (2013), "La segregación escolar en nuestro sistema educativo", Forum Aragón, núm. 10, pp. 47-52.

Sistema de Información de Tendencias Educativas en América Latina (SITEAL) (2021), "Indicadores estadísticos”, en: https://siteal.iiep.unesco.org /pais/ (consulta: 20 de mayo de 2021).

Valenzuela, Juan Pablo, Cristián Bellei y Danae de los Ríos (2010), "Segregación escolar en Chile", en Sergio Martinic y Gregory Elacqua (eds.), ¿Fin de ciclo? Cambios en la gobernanza del sistema educativo chileno, Santiago de Chile, UNESCO-OREALC, pp. 257-284.

Verger, Antoni, Mauro Moschetti y Clara Fontdevila (2017), La privatización educativa en América Latina: una cartografía de políticas, tendencias y trayectorias, Bruselas, La Internacional de la Educación. 